\title{
ARTICLE OPEN \\ Highly coherent spin states in carbon nanotubes coupled to cavity photons
}

\author{
T. Cubaynes ${ }^{1}$, M. R. Delbecq ${ }^{1}{ }^{1}$, M. C. Dartiailh ${ }^{1}$, R. Assouly ${ }^{1}$, M. M. Desjardins ${ }^{1}$, L. C. Contamin ${ }^{1}$, L. E. Bruhat ${ }^{1,2}$, Z. Leghtas ${ }^{1,3,4}$, F. Mallet ${ }^{1}$, \\ A. Cottet ${ }^{1}$ and T. Kontos ${ }^{1}$
}

Spins confined in quantum dots are considered as a promising platform for quantum information processing. While many advanced quantum operations have been demonstrated, experimental as well as theoretical efforts are now focusing on the development of scalable spin quantum bit architectures. One particularly promising method relies on the coupling of spin quantum bits to microwave cavity photons. This would enable the coupling of distant spins via the exchange of virtual photons for two qubit gate applications, which still remains to be demonstrated with spin qubits. Here, we use a circuit QED spin-photon interface to drive a single electronic spin in a carbon nanotube-based double quantum dot using cavity photons. The microwave spectroscopy allows us to identify an electrically controlled spin transition with a decoherence rate which can be tuned to be as low as $250 \mathrm{kHz}$. We show that this value is consistent with the expected hyperfine coupling in carbon nanotubes. These coherence properties, which can be attributed to the use of pristine carbon nanotubes stapled inside the cavity, should enable coherent spin-spin interaction via cavity photons and compare favorably to the ones recently demonstrated in Si-based circuit QED experiments. Our clean and controlled nano-assembly technique of carbon nanotubes in the cavity could be further improved by purified ${ }^{12} \mathrm{C}$ growth to get rid of the nuclear spins resulting in an even higher spin coherence.

npj Quantum Information (2019)5:47; https://doi.org/10.1038/s41534-019-0169-4

\section{INTRODUCTION}

The observation of strong coupling between the charge or the spin confined in a quantum dot circuit and cavity photons has been reported very recently, ${ }^{1-6}$ bringing closer the demonstration of distant single spin-single spin interaction, ${ }^{7-13}$ in the quest for scalable quantum information processing platforms. ${ }^{14}$ So far, such a distant spin coupling mediated by cavity photons has been achieved only for large spin ensembles. ${ }^{15,16}$ One critical parameter of a spin-photon platform is the linewidth of the spin transition which sets the maximum number of coherent swap operations between a single spin and a photon. Whereas they are well documented in $\mathrm{Si}$ or GaAs, the coherence properties of single electronic spins in carbon nanotubes are still debated. ${ }^{17,18}$

Here, we use a spin qubit scheme based on a carbon nanotube embedded in a microwave cavity. Our device is made using a stapling technique developed for CQED architectures, which produces ultraclean double quantum dot devices with near-ideal spectra. We use the circuit QED platform to perform a microwave spectroscopy of the spin transition. We observe the characteristic dispersion of the spin transition of our spin qubit. When the qubit states are tuned to be almost pure spin states (see Fig. 1d), the measured decoherence rate is found to be as low as $250 \mathrm{kHz}$. Strikingly, such a figure of merit is more than 100 times better than in previous work on carbon nanotubes ${ }^{17,18}$ and compares favorably to the very recent values reported for Si-based devices in a circuit QED environment. ${ }^{4,5}$ From the gate dependence of the decoherence rate, we show that the charge noise is the main source of decoherence for the spin when the qubit states are mixed charge/spin states, but that it can be substantially reduced in the spin qubit regime.

The principle of our spin-photon coupling relies on two noncollinear Zeeman fields on each quantum dots (see Fig. 1c, e) in a double quantum dot, originating from zig-zag shaped ferromagnetic contacts. These non-collinear Zeeman fields can be achieved by interface exchange fields ${ }^{8}$ or by stray magnetic fields ${ }^{9,19}$ which both give the same Hamiltonian. In our case, the interface exchange fields a priori dominate. ${ }^{20}$ In the adiabatic regime, if an electronic spin is located on the left dot, it aligns along the left spin quantization axis whereas if it is located on the right dot, it aligns along the right spin quantization axis. Since the two dots are separated by few hundreds of $\mathrm{nm}$, there is a large (mesoscopic) electric dipole between the left and the right dots which is given to the spin thanks to the non-collinear magnetizations. The photons of the cavity convey an electrical field which couples to this electric dipole and therefore to the spin. An alternative wording is to state that the ferromagnetic electrodes give rise to a two-site artificial spin-orbit coupling, which makes the spin sensitive to the cavity electric field. ${ }^{20}$ It is interesting to note that such an "orbitally" mediated spin-photon coupling allows one to increase the natural spin-photon coupling by about 5 orders of magnitude ${ }^{4,5,20}$ without degrading substantially the inherent good coherence properties of a single spin if the device is used in the limit where the electron is trapped almost completely in one of the two dots (left or right). This regime can be reached by detuning the left dot orbital energy $\varepsilon_{\mathrm{L}}$ (tunable with gate

\footnotetext{
'Laboratoire de Physique de I'Ecole Normale Supérieure, ENS, Université PSL, CNRS, Sorbonne Université, Université Paris-Diderot, Sorbonne Paris Cité, Paris, France;

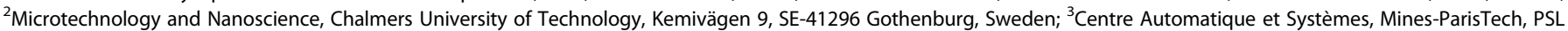
Research University, 60 bd Saint-Michel, 75006 Paris, France and ${ }^{4}$ QUANTIC Team, INRIA de Paris, 2 Rue Simone Iff, 75012 Paris, France Correspondence: A Cottet (cottet@lpa.ens.fr) or T Kontos (kontos@lpa.ens.fr)
}

Received: 9 January 2019 Accepted: 7 June 2019

Published online: 08 July 2019 
(a)

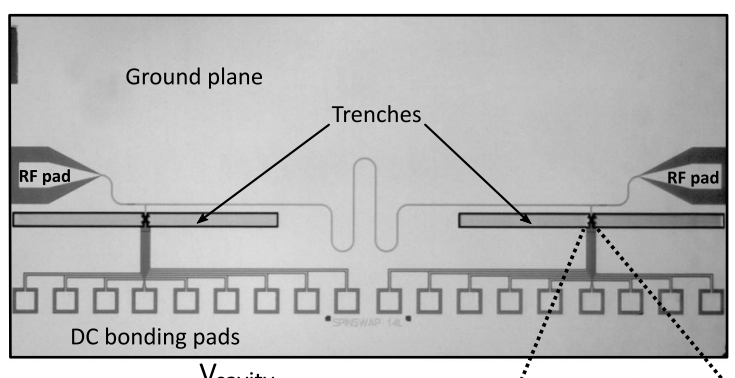

(c)
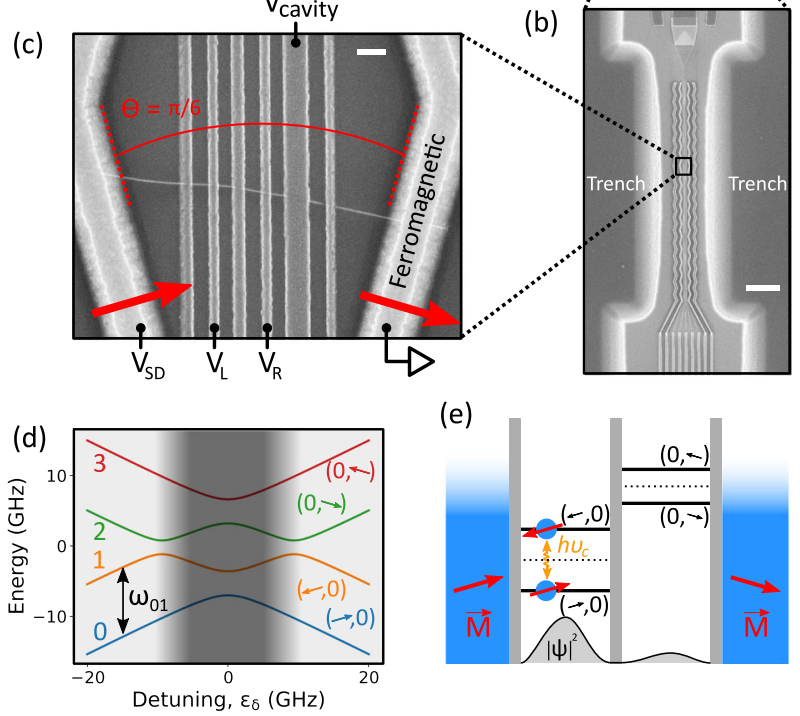

(e)

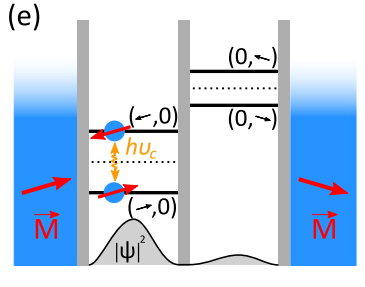

Fig. 1 Principle of spin-photon interface. a Large scale view of our circuit QED setup. Only one of two circuit areas is used. Scale bar: $1 \mathrm{~mm}$. b Scanning electron microscope (SEM) picture of the pedestal structure on which the device is made with the zig-zag magnetic contacts. Scale bar: $10 \mu \mathrm{m}$. c Zoom on the device showing the bottom gates and the non-collinear ferromagnetic contacts. Scale bar: $200 \mathrm{~nm}$. d Spectrum of our spin quantum bit. The spin transition addressed in this work, the 01 transition, saturates to a value defined by the effective Zeeman splitting of each dot at large detuning. In the shaded gray region, spin and charge are not good quantum numbers anymore. e Schematics of our device showing the concept of spin-photon coupling

voltage $V_{L}$ ) from the right dot orbital energy $\varepsilon_{R}$ (tunable with the gate voltage $V_{R}$ ). In such a gate configuration, the electron is trapped almost completely in one of the two dots, as sketched in Fig. 1e. This allows to define the detuning $\varepsilon_{\delta}=a_{\mathrm{L}} V_{\mathrm{L}}-a_{\mathrm{R}} V_{\mathrm{R}}+\varepsilon_{0}$ along the white arrow of Fig. $2 \mathrm{~b}, a_{\mathrm{L}(\mathrm{R})}$ being given by the slope of the degeneracy line. At the large detuning working point, the spin qubit is nearly insensitive to charge noise because of the almost flat energy dispersion but it keeps a coupling to the cavity field larger than the spin decoherence rate. ${ }^{8,19}$

\section{RESULTS}

In the double quantum dot regime, each ferromagnetic contact in our device polarizes one quantum dot. This generates the spectrum depicted in Fig. 1d. ${ }^{8,19}$ Each $K / K^{\prime}$ valleys of carbon nanotubes have a similar spectrum and we omit the valley index here. In addition, we study transitions outside the shaded gray region where the electronic states become pure spin states as the detuning is increased. Several features indicate very weak disorder and electrostatic control of the potential landscape of confined electrons via the bottom gates: a clear "electron-electron quadrant" delimited by the semiconducting gap controlled by two of the bottom gates $\left(V_{L}\right.$ and $\left.V_{R}\right)$, continuous transition from double-dot spectrum (triple points and avoided crossings) to single dot spectrum (parallel transverse lines), and rather regularly spaced Coulomb blockade peaks. At the edges of the electron-electron quadrant of the stability diagram, we can form a double quantum dot in a controlled way in the few electron regime. We estimate the number of electrons $N \approx 10 \pm 5$. However, it is typically only the last electron added to the nanotube which interacts electrically with the cavity. We focus here on the zero detuning line between two charge states highlighted by a white square in Fig. 2a. The phase contrast of the microwave signal in this region is displayed in Fig. 2b. The phase of the microwave signal displays the characteristic sign change of a resonant interaction between the cavity and the double quantum dot. A transition to the dispersive (off-resonant) regime is also visible by a gradual change of phase contrast along this degeneracy line. In the resonant regime, the dependence of the phase contrast $\Delta \phi$ as a function of $\varepsilon_{\delta}$ which has maxima/minima of about $\pm 15^{\circ}$ provides an estimate of the charge coupling strength $g_{\mathrm{C}} \approx 2 \pi(21 \pm 1) \mathrm{MHz}$ and of the charge decay rate $\gamma_{\mathrm{C}} \approx 2 \pi(1.35 \pm 0.16) \mathrm{GHz}$ (see Supplementary Information).

The measured phase is determined by an average of the dispersive shifts induced by each transition, weighted by the steady-state occupation of each state (see Fig. 2b) (see e.g., refs. ${ }^{21,22}$ ). Applying a second tone allows to individually address the different transitions, and to recover their respective coupling strength to cavity photons. The microwave spectroscopy of our ferromagnetic spin qubit is conveniently done by reading out in the dispersive regime the phase $\phi$ of the cavity signal when a second tone is applied through the cavity and its frequency is swept. At large detuning $\varepsilon_{\delta}$, the phase is mainly sensitive to the expectation value $\left\langle\sigma_{z}\right\rangle$ of the spin projection along the quantization axis of the left(right) dot. In the dispersive regime, the expression of the phase $\phi$ reads: $\phi=\frac{2 g_{S}^{2}}{k \Delta}\left\langle\sigma_{z}\right\rangle+\phi_{0}$, where $\phi_{0}$ is a constant which only depends on the microwave setup, $k$ is the linewidth of the cavity, $g_{\mathrm{s}}$ is the spin-photon coupling strength and $\Delta=f_{\text {cav }}-f_{\text {spin }}$ is the detuning between the cavity frequency $f_{\text {cav }}$ and the spin qubit frequency $f_{\text {spin. }}$. Such a measurement is shown in Fig. 3a which displays the phase contrast $\Delta \phi$ as a function of the tone frequency $f_{\text {pump }}$ and $\varepsilon_{\delta}$. In order to avoid cavity photon back-action on the spin qubit, we use a pulsed microwave spectroscopy with the pulse sequence shown in Fig. 3c. The qubit is first driven for $t=3 \mu \mathrm{s}$, then the cavity is filled after $90 \mathrm{~ns}$ and finally read-out using a fast data acquisition card for $t=$ $700 \mathrm{~ns}$. Apart from the frequency independent vertical blue stripe which simply signals the left/right degeneracy line at zero detuning, we observe three resonances which disperse close to zero detuning and saturate at $6.506,6.530$, and $6.540 \mathrm{GHz}$, respectively. The dispersion of each of these transitions with a minimum at zero detuning and a saturation at large detuning is characteristic of a transition which becomes a pure spin transition in the large detuning limit due to the perfect localization of the electron in one quantum dot (see Fig. 1d). The saturation value is given by the effective Zeeman field felt by the (pure) spin state at large detuning. The fact that we observe several spin transitions can be attributed to the lifting of the $K / K^{\prime}$ valley degeneracy of the nanotube as well as from the fact that we are not in the single electron regime. As expected for a spin transition, we can tune the value of this saturation with the external magnetic field. The resulting dependence is shown in Fig. $3 \mathrm{~b}$. The low slope is consistent with previous measurements in a similar architecture with non-stapled nanotube material ${ }^{20}$ and could arise from field modulated exchange coupling between the dot's spin and its adjacent ferromagnetic contact ${ }^{23}$ (see Supplementary Information).

A cut along the lowest resonance at large detuning is shown in Fig. $3 d$. This measurement fitted by a Lorentzian has a full width at half maximum of $\gamma_{\mathrm{FWHM}}=2 \pi \times(498 \pm 80) \mathrm{kHz}$ which sets an upper bound of the decoherence rate $\gamma_{\mathrm{S}} \leq \gamma_{\mathrm{FWHM}} / 2=2 \pi \times 249 \mathrm{kHz}$. Such a narrow line width is 2 orders of magnitude lower than that 


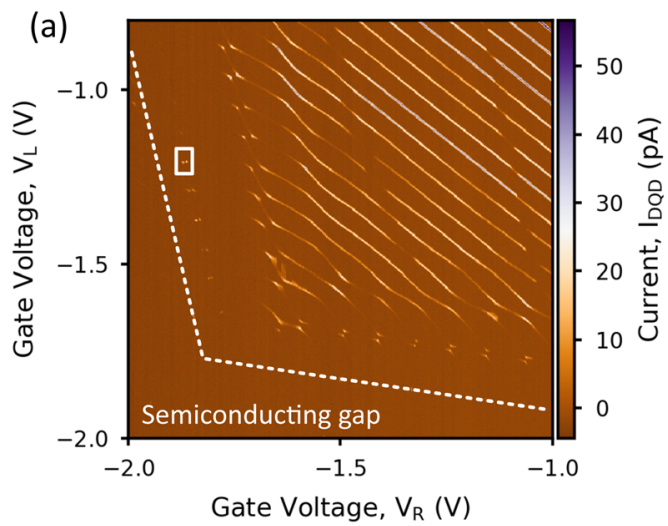

(b)

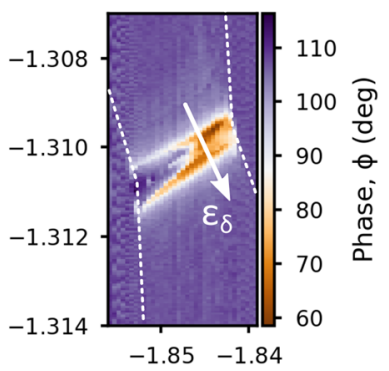

Gate Voltage, $V_{R}(\mathrm{~V})$

Gate Voltage, $V_{R}(V)$

Fig. 2 Carbon nanotube double quantum dot in the few electron regime. a Stability diagram of our double quantum dot spin qubit, given by the current $/$ through the double dot versus the gate voltages $V_{L}$ and $V_{R}$. The gap is at the lower left corner of the stability diagram and is indicated by white dashed lines. $\mathbf{b}$ Phase contrast for the transmitted microwave signal along a charge degeneracy line in the region indicated by the white square in (a)

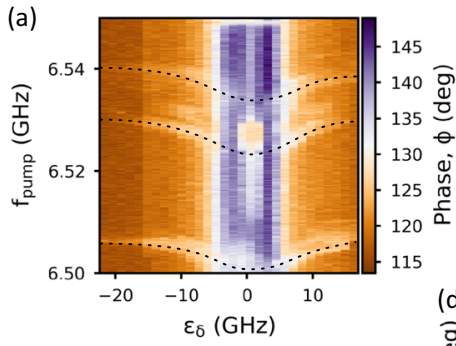

(c)

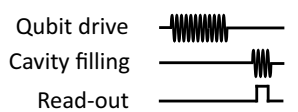

(b)

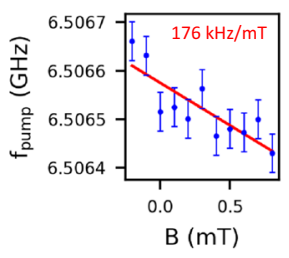

कृ

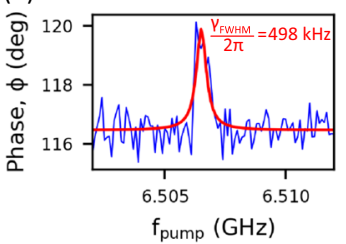

Fig. 3 Microwave spectroscopy of spin transition. a Microwave twotone spectroscopy. b Magnetic field dependence of the microwave frequency (because of setup constraints, the magnetic field span was limited to $\pm 1 \mathrm{mT}$ in this experiment). The error bars are the standard deviation extracted from least square fitting of the spin spectroscopic line in panel (a). c Pulse sequence used for the twotone spectroscopy. d Resonance corresponding to the lowest transition of (a) at a large detuning $<-21 \mathrm{GHz}$ (blue line). We cannot give the exact detuning value due to a gate offset jump which occurred during the measurement. The red line is the Lorentzian fit which allows us to extract $\gamma_{\mathrm{FWHM}}$

found in the valley-spin qubit in the previous work with carbon nanotubes ${ }^{18,24}$ and compares favorably to the very recent figures of merit reported for Si- or GaAs-based platforms. ${ }^{4-6}$ Specifically, our decoherence rate is about 2-4 times lower than the Si-based platforms and about 40 times lower than the GaAs platform. We speculate that the figure of merit of our material is mainly due to the purity of our nanotube-metal interfaces. In addition, our fabrication technique provides a priori quantum dots far from surface oxide or uncontrolled adsorbates on surfaces which are known sources of dephasing. From the phase contrast of about $4^{\circ}$, we can estimate a lower bound spin-photon coupling strength $g_{\mathrm{s}} \approx 2 \pi \times(2.0 \pm 0.1) \mathrm{MHz}$ (see Supplementary Information), which exceeds the decoherence rate of the spin states (and of the cavity) and therefore implies that the spin is strongly coupled to the cavity photons although they are not resonant. As a comparison, we have $g_{\mathrm{S}} / \gamma_{\mathrm{S}} \geq 8$ for the spin transition whereas $g_{\mathrm{C}} / \gamma_{\mathrm{C}} \approx 0.015$ for the charge like transition (see Supplementary Information). There is therefore a very large gain in the coherence properties of our

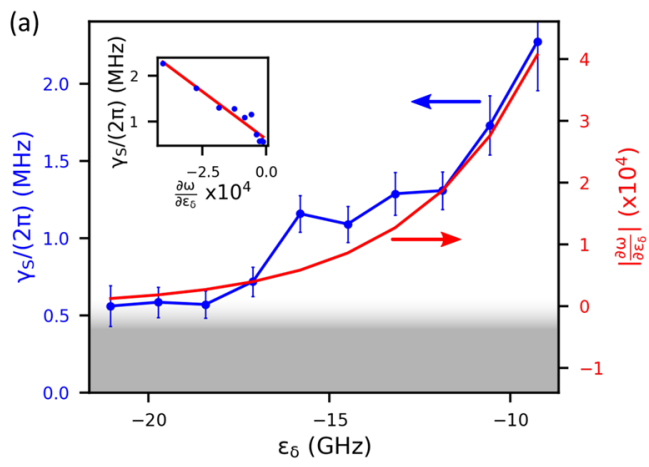

(b)
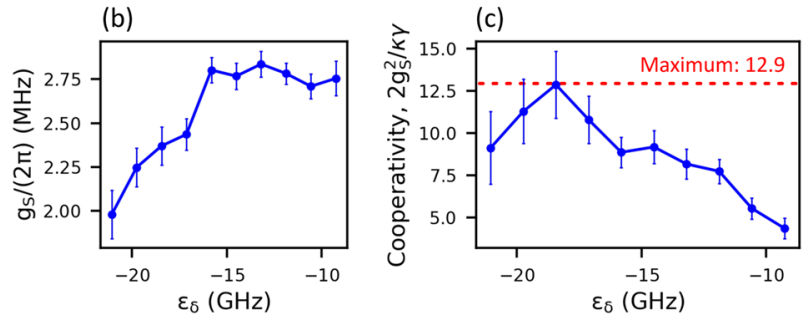

Fig. 4 Nuclear spin limited spin-photon interface. a Linewidth and derivative of the dispersion relation of the spin transition as a function of detuning (the derivative is obtained by fitting the dispersion relation of Fig. $3 a$ and calculating the derivative from the fit). A constant corresponding to a decoherence rate of $560 \mathrm{kHz}$ is added to the derivative. Inset: Linewidth as a function of derivative. b Spin-photon coupling strength as a function of detuning. c Spin-photon cooperativity as a function of detuning. The error bars are the standard deviation extracted from least square fitting of the spin spectroscopic line in Fig. 3a

device when we switch from the charge like transitions to the spin transitions.

\section{DISCUSSION}

In order to specify the decoherence mechanism explaining the linewidth found for our spin transition, we have measured the dependence of the decoherence rate as a function of the detuning $\varepsilon_{\delta}$. Such a measurement is displayed in Fig. 4a. Two main decoherence sources are expected for the electronic spin in double quantum dots: charge noise and nuclear spin. Our ${ }^{12} \mathrm{C}$ platform is grown from a natural $\mathrm{CH}_{4}$ feedstock gas and is thus expected to have a low concentration of nuclear spins $(1.1 \%$ of 
$\left.{ }^{13} \mathrm{C}\right)$. The charge noise is related to the fact that the qubit transition frequency may fluctuate if offset charges nearby the device change the detuning. Therefore, it should induce a decoherence rate $\gamma_{\mathrm{s}}$ proportional to the derivative of the qubit transition frequency with respect to the detuning. ${ }^{8}$ For a large detuning $\varepsilon_{\delta}$, the nuclear spin bath is on the contrary expected to give a nearly independent contribution as a function of the detuning. The decoherence rate $\gamma_{\mathrm{s}}$ and the derivative $\partial \omega / \partial \varepsilon_{\delta}$ as a function of detuning $\varepsilon_{\delta}$ are shown to overlap well provided we add a residual constant of about $500 \mathrm{kHz}$ to the derivative in Fig. 4a. The linear behavior of the decoherence rate $\gamma_{\mathrm{s}}$ as a function of the derivative $\partial \omega / \partial \varepsilon_{\delta}$, displayed in inset, shows that our spin-photon interface is dominated by charge noise at small detuning. Interestingly, it allows us from the slope of the linear behavior to extract a charge noise detuning variance of about $34 \mu \mathrm{eV}$. While this noise is larger than in the previous work in carbon nanotubes $^{22}$ and could be in principle easily lowered, it is interesting to see that we can completely reduce its influence by going at large detuning while keeping a large spin-photon coupling strength with respect to $\gamma_{\mathrm{s}}$. The shaded gray corresponds to the residual decoherence mechanisms with a decoherence rate in the range $\approx 2 \pi \times 500 \mathrm{kHz}$. Note that this value corresponds to twice as much as the lowest decoherence rate presented in Fig. 3d $(250 \mathrm{kHz})$, probably because it corresponds to a lower detuning. Interestingly, the residual decoherence rate allows us to give an upper bound of the contribution of the nuclear spins of the $1.1 \%$ of ${ }^{13} \mathrm{C}$ and therefore of the hyperfine coupling $\mathcal{A}$. From the estimated diameter of our chemical vapor deposition (CVD) nanotubes $D \approx 2 \mathrm{~nm}$ and the length of each dot $d \approx 500 \mathrm{~nm}$, we get a number of nuclear spins of $N \approx 0.011 \times 3 \times 10^{5}$ which yields $\gamma_{\mathrm{s}} \approx 2 \pi \times 200 \mathrm{kHz}$ if $\mathcal{A}=0.1 \mu \mathrm{eV}$. Our measurements are therefore fully in agreement with the tabulated values for the hyperfine coupling expected in CNTs of $\mathcal{A} \approx 0.1-0.5 \mu \mathrm{eV}$ (see ref. ${ }^{18}$ and reference therein). In addition to the decoherence rate, we also present the spin-photon coupling strength and the cooperativity of the spin-photon interface $\left(C=\left(2 g_{\mathrm{S}}^{2}\right) /\left(\kappa \times \gamma_{\mathrm{S}}\right)\right)$ as a function of the detuning (Fig. 4b, $c$, respectively). Interestingly this last quantity allows to identify an optimal detuning working point around $\varepsilon_{\delta}=-18 \mathrm{GHz}$. For this detuning, the hybridization with the charge creates a sizeable spin-photon coupling while maintaining a low decoherence rate. ${ }^{4,5}$

In summary, we have demonstrated that carbon nanotubebased double quantum dots can provide a tunable and coherent spin-photon interface. The figures of merit of coupling strength of $g_{\mathrm{s}} \approx 2 \pi \times 2.0 \mathrm{MHz}$ and low decoherence rate $\gamma_{\mathrm{s}} \approx 2 \pi \times 250 \mathrm{kHz}$ are suitable for future swap experiments. It could be interesting in that context to increase the value of $g_{\mathrm{s}}$. We anticipate that this could be done by optimizing the angle between the ferromagnets ${ }^{8,19}$ or by increasing the impedance of our $50 \Omega$ resonator similarly to refs. ${ }^{4-6}$ In principle, our clean and controlled nanoassembly technique of carbon nanotubes in the cavity could result in even lower spin decoherence rate by using purified ${ }^{12} \mathrm{C}$ in the growth to get rid of the nuclear spins. In addition, since it naturally provides suspended carbon nanotubes, our platform tends to enhance the relaxation time $T_{1}$ of the spin by inhibition of spontaneous emission of phonons (inverse of the Purcell effect). ${ }^{8}$ We could further exploit this effect in future experiments. Our results suggest that carbon, like silicon, can be a promising host for electronic spins encoding quantum information.

\section{METHODS}

Our devices are made with a complete dry transfer nanotube technique adapted from previous works ${ }^{24-28}$ which allows us to integrate as-grown carbon nanotubes in a microwave cavity. The full chip, comprising the cavity, the bottom gates, and the non-collinear ferromagnetic contacts shown in Fig. 1a-c respectively, is placed in a vacuum chamber with a base pressure of $5 \times 10^{-7} \mathrm{mbar}$. The zig-zag contacts visible in Fig. $1 \mathrm{~b}$ and partially in Fig. 1c are NiPd ferromagnetic contacts with transverse magnetization. ${ }^{20}$ Carbon nanotubes are grown on a $\mathrm{Si}$ comb with a standard CVD recipe with $\mathrm{CH}_{4}$ as feedstock gas. The comb is mounted inside the chamber on a stage with micro- and nano-manipulators which allow us to place the nanotube on the chip with controlled approach steps of about $100 \mathrm{~nm}$. The assembly of the carbon nanotube and the ferromagnetic contacts is done under vacuum at a pressure of about $1 \times 10^{-6} \mathrm{mbar}$ in order to ensure a clean interface between the nanotube and the metallic contacts. This results in the device shown in Fig. 1c where a nanotube bridges the two ferromagnetic contacts and is a priori suspended over bottom gates. The wider gate visible in the SEM picture of Fig. $1 \mathrm{c}$ is galvanically coupled to the central conductor of the $\mathrm{Nb}$ cavity visible in Fig. 1a. The cavity fundamental resonance frequency is $6.424 \mathrm{GHz}$ and its quality factor is about 4200 .

The devices obtained with our fabrication technique are more tunable than the previous nanotube-based spin quantum bits and much less disordered. ${ }^{20}$ The measurement setup is similar to the setup of ref. ${ }^{20} \mathrm{We}$ measure simultaneously the DC current I flowing through the device and the microwave signal transmitted through the cavity in amplitude $A$ and phase $\phi$. The control which we have on the spectrum of the device is visible from the stability diagram shown in Fig. 2a which displays the current under a bias of $V_{s d}=100 \mu \mathrm{V}$. The horizontal axis of the microwave spectroscopy is calibrated using the triangles observed in the transport data (not shown). The calibrated parameters are the charging energies $E_{\mathrm{CR}}$ $=2.36 \mathrm{meV}, E_{\mathrm{cL}}=2.21 \mathrm{meV}, U_{\mathrm{m}}=0.61 \mathrm{meV}$ and the gate capacitances $C_{\mathrm{gL}}$ $=1.6 \mathrm{aF}, \mathrm{C}_{\mathrm{gR}}=0.9 \mathrm{aF}$.

\section{DATA AVAILABILITY}

The datasets generated during and/or analyzed during the current study are available from the corresponding authors on reasonable request.

\section{ACKNOWLEDGEMENTS}

The devices have been made within the consortium Salle Blanche Paris Centre. The authors gratefully acknowledge help from José Palomo, Aurélie Pierret, and Michael Rosticher. This work is supported by the ERC Starting Grant "CirQys" and by the ANR "FunTheme".

\section{AUTHOR CONTRIBUTIONS}

T.C. performed the measurements with the help of R.A., M.R.D. and T.K. T.C. developed the stapling technique with the help of M.R.D., M.M.D., L.C.C., M.C.D. and T.K. L.E.B. wired the cryostat and developed part of the experimental setup. M.C.D. contributed through early experiments and developed part of the experimental setup. Z.L. and F.M. contributed through their knowledge on microwave measurement and control of qubits. A.C. contributed through theory material. T.K. wrote the manuscript with inputs from T.C., M.R.D., M.M.D., L.C.C., L.E.B., M.C.D., Z.L., F.M. and A.C. The project is based on an idea by A.C. and T.K.

\section{ADDITIONAL INFORMATION}

Supplementary Information accompanies the paper on the npj Quantum Information website (https://doi.org/10.1038/s41534-019-0169-4).

Competing interests: The authors declare no competing interests.

Publisher's note: Springer Nature remains neutral with regard to jurisdictional claims in published maps and institutional affiliations.

\section{REFERENCES}

1. Bruhat, L. E. et al. Circuit QED with a quantum-dot charge qubit dressed by Cooper pairs. Phys. Rev. B 98, 155313 (2018).

2. Mi, X., Cady, J. V., Zajac, D. M., Deelman, P. W. \& Petta, J. R. Strong coupling of a single electron in silicon to a microwave photon. Science 355, 156-158 (2017).

3. Stockklauser, A. et al. Strong coupling cavity QED with gate-defined double quantum dots enabled by a high impedance resonator. Phys. Rev. X 7, 011030 (2017).

4. $\mathrm{Mi}, \mathrm{X}$. et al. A coherent spin-photon interface in silicon. Nature 555, 599-603 (2018).

5. Samkharadze, N. et al. Strong spin-photon coupling in silicon. Science 405, 1123-1127 (2018). 
6. Landig, A. J. et al. Coherent spin-photon coupling using a resonant exchange qubit. Nature 560, 179-184 (2018).

7. Trif, M., Golovach, V. N. \& Loss, D. Spin dynamics in InAs nanowire quantum dots coupled to a transmission line. Phys. Rev. B 77, 045434 (2008).

8. Cottet, A. \& Kontos, T. A spin quantum bit with ferromagnetic contacts for circuit QED. Phys. Rev. Lett. 105, 160502 (2010).

9. Hu, X., Liu, Y.-X. \& Nori, F. Strong coupling of a spin qubit to a superconducting stripline cavity. Phys. Rev. B 86, 035314 (2012).

10. Harvey, S. P. et al. Coupling two spin qubits with a high-impedance resonator. Phys. Rev. B 97, 235409 (2018).

11. Majer, J. et al. Coupling superconducting qubits via a cavity bus. Nature 449 , 443-447 (2007).

12. Sillanpää, M. A., Park, J. I. \& Simmonds, R. W. Coherent quantum state storage and transfer between two phase qubits via a resonant cavity. Nature 449, 438 (2007).

13. Blais, A., Huang, R.-S., Wallraff, A., Girvin, S. M. \& Schoelkopf, R. J. Cavity quantum electrodynamics for superconducting electrical circuits: an architecture for quantum computation. Phys. Rev. A 69, 062320 (2004).

14. Loss, D. \& Di Vincenzo, D. Quantum computation with quantum dots. Phys. Rev. A 61, 2472 (1998).

15. Ghirri, A. et al. Coherently coupling distinct spin ensembles through a high- $T_{c}$ superconducting resonator. Phys. Rev. A 93, 063855 (2016).

16. Astner, T. et al. Coherent coupling of remote spin ensembles via a cavity bus. Phys. Rev. Lett. 118, 140502 (2017).

17. Laird, E. A. et al. Quantum transport in carbon nanotubes. Rev. Mod. Phys. 87, 703 (2015).

18. Pei, T. et al. Hyperfine and spin-orbit coupling effects on decay of spin-valley states in a carbon nanotube. Phys. Rev. Lett. 118, 177701 (2017).

19. Benito, M., Mi, X., Taylor, J. M., Petta, J. R. \& Burkard, G. Input-output theory for spin-photon coupling in Si double quantum dots. Phys. Rev. B 96, 235434 (2017).

20. Viennot, J. J., Dartiailh, M. C., Cottet, A. \& Kontos, T. Coherent coupling of a single spin to microwave cavity photons. Science 349, 408 (2015).

21. Petersson, K. D. et al. Circuit QED with a spin orbit qubit. Nature 490, 380-383 (2012)
22. Viennot, J. J., Delbecq, M. R., Dartiailh, M. C., Cottet, A. \& Kontos, T. Out of equilibirum charge dynamics in a circuit QED architecture. Phys. Rev. B 89, 165404 (2014)

23. Cottet, A. Gate-dependent spin-torque in a nanoconductor-based spin-valve. Phys. Rev. B 84, 054402 (2011).

24. Laird, E. A., Pei, F. \& Kouwenhoven, L. P. A valley-spin qubit in a carbon nanotube. Nat. Nanotechnol. 8, 565-568 (2013).

25. Wu, C. C., Liu, C. H. \& Zhong, Z. One-step direct transfer of pristine single-walled carbon nanotubes for functional nanoelectronics. Nano Lett. 10, 1032-1036 (2010).

26. Waissman, J. et al. Realization of pristine and locally tunable one-dimensional electron systems in carbon nanotubes. Nat. Nanotechnol. 8, 569-574 (2013).

27. Viennot, J. J., Palomo, J. \& Kontos, T. Stamping carbon nanotubes for circuit QED. Appl. Phys. Lett. 104, 113108 (2014).

28. Ranjan, V. et al. Clean carbon nanotubes coupled to superconducting impedance-matching circuits. Nat. Commun. 6, 7165 (2015).

(i) Open Access This article is licensed under a Creative Commons Attribution 4.0 International License, which permits use, sharing, adaptation, distribution and reproduction in any medium or format, as long as you give appropriate credit to the original author(s) and the source, provide a link to the Creative Commons license, and indicate if changes were made. The images or other third party material in this article are included in the article's Creative Commons license, unless indicated otherwise in a credit line to the material. If material is not included in the article's Creative Commons license and your intended use is not permitted by statutory regulation or exceeds the permitted use, you will need to obtain permission directly from the copyright holder. To view a copy of this license, visit http://creativecommons. org/licenses/by/4.0/.

C The Author(s) 2019 